\title{
Nutlin-3 sensitizes nasopharyngeal carcinoma cells to cisplatin-induced cytotoxicity
}

\author{
YEE-LIN VOON $^{1,2}$, MUNIRAH AHMAD ${ }^{1}$, POOI-FONG WONG ${ }^{2}$, ROSLINA HUSAINI ${ }^{1}$, \\ WAYNE TIONG-WENG NG ${ }^{1}$, CHEE-ONN LEONG ${ }^{3}$, DAVID PHILIP LANE ${ }^{4}$ and ALAN SOO-BENG KHOO ${ }^{1}$ \\ ${ }^{1}$ Molecular Pathology Unit, Cancer Research Centre, Institute for Medical Research, Kuala Lumpur 50588; \\ ${ }^{2}$ Department of Pharmacology, Faculty of Medicine, University of Malaya, Kuala Lumpur 50603; \\ ${ }^{3}$ School of Pharmacy and Health Sciences, International Medical University, Kuala Lumpur 57000, Malaysia; \\ ${ }^{4}$ p53 Laboratory (p53Lab), Agency for Science, Technology and Research (A*STAR), \\ 8A Biomedical Grove, Immunos, Singapore 138648, Republic of Singapore
}

Received April 24, 2015; Accepted June 29, 2015

DOI: 10.3892/or.2015.4177

\begin{abstract}
The small-molecule inhibitor of p53-Mdm2 interaction, Nutlin-3, is known to be effective against cancers expressing wild-type (wt) p53. p53 mutations are rare in nasopharyngeal carcinoma (NPC), hence targeting disruption of p53-Mdm2 interaction to reactivate p53 may offer a promising therapeutic strategy for NPC. In the present study, the effects of Nutlin-3 alone or in combination with cisplatin, a standard chemotherapeutic agent, were tested on C666-1 cells, an Epstein-Barr virus (EBV)-positive NPC cell line bearing wt p53. Treatment with Nutlin-3 activated the p53 pathway and sensitized NPC cells to the cytotoxic effects of cisplatin. The combined treatment also markedly suppressed soft agar colony growth formation and increased apoptosis of NPC cells. The effect of Nutlin-3 on NPC cells was inhibited by knockdown of p53, suggesting that its effect was p53-dependent. Extended treatment with increasing concentrations of Nutlin-3 did not result in emergence of p53 mutations in the C666-1 cells. Collectively, the present study revealed supportive evidence of the effectiveness of combining cisplatin and Nutlin-3 as a potential therapy against NPC.
\end{abstract}

\section{Introduction}

Nasopharyngeal carcinoma (NPC) is a common epithelial squamous-cell head and neck carcinoma, which originates from the nasopharyngeal mucosa and is associated with Epstein-Barr virus (EBV) infection (1). This type of cancer

Correspondence to: Dr Alan Soo-Beng Khoo, Molecular Pathology Unit, Cancer Research Centre, Institute for Medical Research, Jalan Pahang, Kuala Lumpur 50588, Malaysia

E-mail: alankhoo@imr.gov.my

Key words: apoptosis, C666-1 cells, drug resistance, p53 knockdown, p53-Mdm2 targeted therapy, synergism, Nutlin-3 shows a distinct racial and geographical distribution and is prevalent among Southern Chinese, as well as natives in Southeast Asia including Malaysia $(2,3)$. In the early stages, NPC may be asymptomatic or present with apparently trivial symptoms and is therefore likely to be ignored resulting in late diagnosis $(4,5)$.

NPC is usually treated with radiotherapy and/or chemotherapy (cisplatin and 5-fluorouracil) (6). Concurrent chemo-radiotherapy is the main modality of treatment for advanced-stage NPC (7). However, advanced-stage NPC is associated with poor prognosis and therapeutic failure (7). Recurrence, distant metastases, treatment resistance and adverse effects of treatment remain the major challenges in the treatment of NPC (8-10).

p53, a tumor suppressor gene, is a transcription factor which controls genes involved in DNA repair, cell cycle arrest and apoptosis (11). Murine double minute (Mdm2) is an important negative regulator of p53 (12). It is an E3 ubiquitin ligase which promotes degradation of $\mathrm{p} 53$ via the ubiquitin-proteosome pathway $(13,14)$ and functions in an autoregulatory feedback loop with p53. Inhibition of the p53-Mdm2 interaction prevents this degradation, thus resulting in increased $\mathrm{p} 53$ levels.

Nutlins are cis-imidazoline analogs (14) which compete with Mdm 2 for binding to $\mathrm{p} 53$. Nutlin-3 has been reported to be effective in killing cancer cells retaining functional wt p53 (15). Nutlin-3 exerts anticancer effects on acute myeloid (16) and chronic lymphocytic leukemia (17), multiple myeloma (18), Kaposi sarcoma (19), liposarcoma (20), rhabdomyosarcoma (21), Ewing's sarcoma (22), colon (23) and testicular cancer (24), osteosarcoma and other types of cancer (25). Nutlin-3 suppressed not only tumor growth, but also distant metastasis in a xenograft model of wt p53 neuroblastoma (26). Moreover, Nutlin-3 selectively enhanced apoptosis in wt p53 cancer cells by activating the p53 pathway $(16,26)$. Currently, drugs which reactivate the $\mathrm{p} 53$ pathway are undergoing clinical trials (27). Although Nutlin-3 has been reported to be effective against a wide variety of tumors bearing wt $\mathrm{p} 53$, the effects of Nutlin-3 on NPC cells have yet to be reported.

p53 mutations have been reported to be rare in NPC $(28,29)$, even in recurrent radioresistant NPC (30), thus 
making this cancer a potential candidate for treatment with p53-Mdm2 inhibitors, such as Nutlin-3. However, increased staining of p53 protein by immunohistochemistry has been reported, suggesting the deregulation of the p53 pathway in NPC $(29,31,32)$. In addition, latent genes of EBV, such as LMP1 which is known to be expressed in NPC, has been reported to inhibit the p53 pathway (33). Interestingly, it has been suggested that induced overexpression of p53 using an adenoviral vector was found to be effective against NPC cells $(34,35)$, indicating that further elevation of the p53 levels using the p53 activator, Nutlin-3, may be effective to further improve NPC treatment.

In the present study, we sought to investigate the effects of Nutlin-3 alone or in combination with cisplatin on an EBV-positive, wt p53, NPC cell line, C666-1. We also tested whether extended treatment with Nutlin-3 would result in the emergence of p53 mutations in NPC cells. The findings of the present study provide further insights for the potential use of Nutlin-3 as an NPC therapeutic agent.

\section{Materials and methods}

In vitro cell culture. C666-1 cells were cultured in Roswell Park Memorial Institute (RPMI)-1640 medium (Gibco Life Technologies, Carlsbad, CA, USA) supplemented with $15 \%$ heat-inactivated fetal calf serum (FCS) (Gibco Life Technologies). EBV-negative NPC HK1 cells were cultured in 10\% FCS RPMI-1640 medium. HCT116, a colorectal carcinoma cell line, and MDA-MB-231, a metastatic breast adenocarcinoma cell line were cultured in Dulbecco's modified Eagle's medium (DMEM) (Gibco Life Technologies) supplemented with $10 \%$ heat-inactivated FCS. Nasopharyngeal epithelial (NPE) cell line, NP69, was cultured in keratinocyte serum-free medium (K-SFM) (Gibco Life Technologies) supplemented with bovine pituitary extract and $0.16 \mathrm{ng} / \mathrm{ml}$ recombinant epidermal growth factor (Gibco Life Technologies), while NPE NP460 cells were maintained in a 1:1 ratio of defined K-SFM medium supplemented with growth factor (Gibco Life Technologies) and EpiLife ${ }^{\circledR}$ Defined Growth Supplement (Cascade Biologics, Life Technologies, Carlsbad, CA, USA). All of the cell lines were maintained in an exponential growth phase in the presence of $10 \mathrm{U} / \mathrm{ml}$ of penicillin (Gibco Life Technologies) and $10 \mu \mathrm{g} / \mathrm{ml}$ streptomycin (Gibco Life Technologies) at $37^{\circ} \mathrm{C}$ in a $5 \% \mathrm{CO}_{2}$ humidified atmosphere. Mycoplasma-free status of the cells was verified monthly with the e-myco ${ }^{\text {TM }}$ Mycoplasma PCR Detection kit (Intron Biotechnology, Inc., Seongnam, Korea).

PCR and DNA sequencing. Genomic DNA was extracted from cultured cells using QIAamp DNA Mini kit (Qiagen, Valencia, CA, USA) following the manufacturer's instructions. p53 gene amplification spanning from exons 2 to 11 was amplified in separate PCR reactions using specific designed oligonucleotide forward (F) and reverse (R) primers synthesized by First BASE Laboratories, Malaysia. Primer sequences used for PCR were: exon 2 (F), 5'-AGC TGT CTC AGA CAC TGG CA-3' and (R), 5'-GAG CAG AAA GTC AGT CCC ATG-3'; exon 3+4 (F), 5'-AGA CCT ATG GAA ACT GTG AGT GGA-3' and (R), 5'-GAA GCC TAA GGG TGA AGA GGA-3'; exon 5 (F), 5'-CGG AAT TCT TAT CTG TTC ACT TGT GCC C-3' and
(R), 5'-CGG GAT CCA CCC TGG GCA ACC AGC CCT G-3'; exon 6 (F), 5'-CGG AAT TCG GTC CCC AGG CCT CTG ATT CCT -3' and (R), 5'-CGG GAT CCA CCC GGA GGG CCA CTG ACA AC-3'; exon 7 (F), 5'-CTG CTT GCC ACA GGT CTC-3' and (R), 5'-TGG ATG GGT AGT AGT ATG GAA G-3'; exon 8 (F), 5'-CGG AAT TCT TGG GAG TAG ATG GAG CCT-3' and (R), 5'-CGG GAT CCC TCC TCC ACC GCT TCT TGT CCT-3'; exon 9 (F), 5'-AGC AAG CAG GAC AAG AAG CG-3' and (R), 5'-CCA GGA GCC ATT GTC TTT GA-3'; exon 10 (F), 5'-CTC AGG TAC TGT GTA TAT ACT TAC-3' and (R), 5'-ATA CTA CGT GGA GGC AAG AAT-3'; exon 11 (F), 5'-TCC CGT TGT CCC AGC GTT-3' and (R), 5'-TAA CCC TTA ACT GCA AGA ACAT-3'. The quality and purity of the resulting PCR products were evaluated on an ethidium bromide stained-agarose gel (2\%). The products were further purified using Qiagen QIA quick PCR Purification kit followed by Sanger sequencing. The data were aligned and compared against the published human p53 sequences in NCBI GenBank (X54156). The nucleotide variants were analyzed using Mutation Surveyor V4.0.7 (SoftGenetics, LLC., State College, PA, USA).

Western blot analysis. Cells were lysed using cold lysis buffer containing $2 \mathrm{mM}$ DL-dithiothreitol, $0.2 \mathrm{mM}$ phenylmethylsulfonylfluoride (Bio-Rad, Hercules, CA, USA) and protease inhibitor cocktail (Roche Diagnostics GmbH, Mannheim, Germany). Total protein lysate $(10 \mu \mathrm{g})$ was separated on $10-15 \%$ sodium dodecyl sulphate-polyacrylamide gel electrophoresis, followed by immunoblotting with monoclonal antibodies for p53 (Promega Corporation, Madison, WI, USA), Mdm2 (Santa Cruz Biotechnology, Inc., Santa Cruz, CA, USA), p21Waf1/Cip1, PUMA, BAX, Bcl2 and PARP (Cell Signaling Technology, Inc., Beverly, MA, USA). $\beta$-actin (Santa Cruz Biotechnology) was used as a loading control and secondary antibody reactions were performed with anti-mouse or anti-rabbit (Promega Corporation) horseradish peroxidase-conjugated $\mathrm{IgG}$. Chemiluminescent imaging was conducted using ImageQuant ${ }^{\mathrm{TM}}$ LAS 500 (GE Healthcare Life Sciences, Buckinghamshire, UK). Densitometric analysis was performed on an Alpha Imager HP System with AlphaView software (Alpha Innotech Corporation, San Leandro, CA, USA).

MTS cell viability assay. Ready-to-use $1 \mathrm{mg} / \mathrm{ml}$ stock cisplatin [cis- $\left.\mathrm{PtCl}_{2}\left(\mathrm{NH}_{3}\right)_{2}\right]$ (Kemoplat, Fresenius Kabi, Maharashtra, India) and Nutlin-3 (Alexis Biochemicals, San Diego, CA, USA) were utilized for the in vitro treatment. Briefly, cells were seeded at densities of $1.5 \times 10^{4}$ cells/ml for NP69 and NP460; and $3 \times 10^{4}$ cells $/ \mathrm{ml}$ for C666-1 cells in 96-well plates (TPP Techno Plastic Products AG, Trasadingen Switzerland). The cells were cultured for $24 \mathrm{~h}$ before treatment. The dose-response curves and half maximal inhibitory concentration $\left(\mathrm{IC}_{50}\right)$ values were determined by $96^{\circledR}$ AQueous One Solution Cell Proliferation MTS solution (Promega Corporation) followed by measurement using an EnVision multilabel plate reader (PerkinElmer, Waltham, MA, USA).

Soft agar colony formation assay. The C666-1 cells were seeded at a density of $5 \times 10^{4}$ cells/ml in 96 -well plates (TPP), followed by cisplatin treatment with or without Nutlin-3. 
The cells were then plated into a two-layer soft agar made from DNA grade Seakem agarose (Lonza, Rockland, ME, USA) culture system (comprised of a layer of $0.3 \%$ agarose in complete media; and with $0.6 \%$ agar as a base layer) in 6-well plates (TPP). Anchorage-independent growth was measured by counting the numbers of viable colonies using an Olympus stereomicroscope model SZX7 (Olympus, Tokyo, Japan). The colonies were scored by using Image Pro Plus AMS version 6.3 (Media Cybernetics, Inc. Rockville, MD, USA). Colonies with a minimum diameter of $60 \mu \mathrm{m}$, area $2,800 \mu \mathrm{m}^{2}$ and roundness score ranging from 0.25 to 0.50 (roundness $=4 \mathrm{~A} / \pi \mathrm{D}^{2} ; \mathrm{A}$ is the area; $\mathrm{D}$ is the maximum diameter; with 1.0 indicating a perfect circle) were counted in order to exclude abortive colonies.

p53 knockdown with small-hairpin RNA (shRNA). p53 knockdown was performed using four lentiviral-based shRNA constructs (Sigma-Aldrich, St. Louis, MO, USA). The shRNA p53-target sequences were as follows: p53si-2 (D3), 5'-CAC CATCCACTACAACTACAT-3'; p53si-3 (C12), 5'-CGGCGC ACAGAGGAAGAGAAT-3'; p53si-4 (E1), 5'-GAGGGATGT TTGGGAGATGTA-3'; p53si-5 (E2), 5'-GTCCAGATGAAG CTCCCAGAA-3' and non-specific (NS), 5'-CAACAAGAT GAAGAGCACCAA-3'. Lentiviral stocks were generated by co-transfecting the HEK-293T cells (ATCC ${ }^{\circledR}$ CRL-3216 ${ }^{\mathrm{TM}}$; American Type Culture Collection, Manassas, VA, USA) with the plasmid vector, the psPAX2 packaging plasmids (Addgene plasmid 12260) and pMD2G envelope plasmid (Addgene plasmid 12259) (Addgene, Inc., Cambridge, MA, USA) using Lipofectamine 2000 (Invitrogen, Life Technologies, Carlsbad, CA, USA) according to the manufacturer's recommendations. The knockdown was verified by western blot analysis.

High content analysis of apoptosis. Briefly, C666-1 cells were seeded at a density of $3 \times 10^{4}$ cells $/ \mathrm{ml}$ in View Plate-96 Black 96-well plates (PerkinElmer) and were allowed to grow for $24 \mathrm{~h}$ prior to cisplatin and/or Nutlin-3 treatments for 48 and $72 \mathrm{~h}$. The cells were cultured with $0.1 \%$ DMSO (Sigma-Aldrich) or basal media, which served as vehicle controls for Nutlin-3 and cisplatin treatments, respectively. The cells were stained with AnnexinV-FITC, propidium iodide (PI) and Hoechst 33342 (BD Biosciences, San Jose, CA, USA) according to the manufacturer's instructions. Well to well imaging with three filter channels (DAPI, FITC and TRITC) was performed using a Metamorph screening acquisition module, on a Nikon Ti-ECLIPSE inverted fluorescence microscope (Nikon Corporation, Tokyo, Japan), at a magnification of x20. Nine fields were imaged and scored for each well using Metamorph software version 7.7.0.0 (Molecular Devices, Downingtown, PA, USA). The percentages of apoptotic cells were calculated from triplicate wells.

Establishment of Nutlin-3-resistant NPC C666-1 cells. Nutlin-3-resistant cells were generated by propagation of parental C666-1 cells in stepwise ascending concentrations $(10,20$ and $40 \mu \mathrm{M})$ of Nutlin-3 for a varying total number of passages (7-36) over a period of up to six months. Cell viability of the Nutlin-3-resistant sublines relative to the control parental C666-1 cells was determined by MTS viability assay after a 72-h treatment with Nutlin-3. The resistance index (R) ( $\mathrm{R}=\mathrm{IC}_{50}$ resistant cells/IC $\mathrm{I}_{50}$ sensitive cells) was calculated to determine the degree of acquired resistance to its relative control parental C666-1 cells.

Statistical analysis. Data were analyzed by Microsoft Excel and/or GraphPad Prism version 5 (GraphPad Software Inc., San Diego, CA, USA). Statistical significance was measured using the Student's paired t-test and P-values $<0.05$ were considered to be statistically significant.

\section{Results}

p53 mutation status of NPC and NPE cells. In the present study, p53 sequences in NPC (C666-1; HK1) and NPE (NP69; NP460) cell lines were first examined by sequencing exons 2 through 11 of the p53 gene. The p53 sequences in C666-1 cells had a single $\mathrm{G}>\mathrm{C}$ base substitution detected at position 12139 at codon 72 of exon 4, while no alteration was detected in other exons (data not shown). An identical alteration was also detected in the NP69 and NP460 cell lines. However, a homozygous $\mathrm{C}>\mathrm{G}$ base substitution at codon 130 of exon 5 was detected in the HK1 cells. Overall, these results suggest that the p53 mutation was only detected in the HK1 cells, while C666-1, NP69 and NP460 cells retained the wt p53 status while expressing a polymorphism at codon 72 , common in the wt p53 gene.

Effects of single drug treatments of cisplatin and Nutlin-3 on NPC and NPE cells. The effects of single drug treatment, cisplatin (Fig. 1A) or Nutlin-3 (Fig. 1B), on the growth and viability of the NPC C666-1 and NPE cell lines were evaluated. The results indicated that both NPC and NPE cells were sensitive to cisplatin and showed clear dose dependence, whereas the NPE cells were less sensitive than the NPC cells to Nutlin-3. The $\mathrm{IC}_{50}$ values showed that cisplatin was more cytotoxic against the NPE cell lines compared to the NPC cells (Table I). In contrast, Nutlin-3 showed stronger cytotoxic effects against the NPC cells compared to the effects in the NPE cells, suggesting that Nutlin-3 may be more selective towards cancer cells.

Combination treatment of cisplatin and Nutlin-3 on NPC cells. The effects of Nutlin-3 in combination with cisplatin on the C666-1 cells were investigated (Fig. 2). Cisplatin alone inhibited the growth of the C666-1 cells in a dose-dependent manner with an $\mathrm{IC}_{50}$ value of $26.55 \pm 6.15 \mu \mathrm{M}$. When C666-1 cells were treated with a combination of cisplatin $(0-100 \mu \mathrm{M})$ and a fixed concentration of $10 \mu \mathrm{M}$ Nutlin-3, the $\mathrm{IC}_{50}$ value was markedly reduced to $3.67 \pm 0.88 \mu \mathrm{M}$ (Fig. 2A). Similarly, when the cells were treated with a combination of Nutlin-3 $(0-30 \mu \mathrm{M})$ and a fixed concentration of $33.33 \mu \mathrm{M}$ cisplatin, the $\mathrm{IC}_{50}$ value of Nutlin-3 was markedly decreased from $10.23 \pm 0.87 \mu \mathrm{M}$ to $0.86 \pm 0.13 \mu \mathrm{M}$ (Fig. $2 \mathrm{~B}$ ), suggesting that the cisplatin combined with Nutlin-3 was more effective than each agent when used separately. These findings provide an early indication that Nutlin-3 sensitizes C666-1 cells to the cytotoxic effects of cisplatin.

Effects of Nutlin-3 on the tumorigenicity of NPC cells in soft agar. The effects of Nutlin-3 in combination with cisplatin on the tumorigenicity of C666-1 cells were assessed by determining 
A

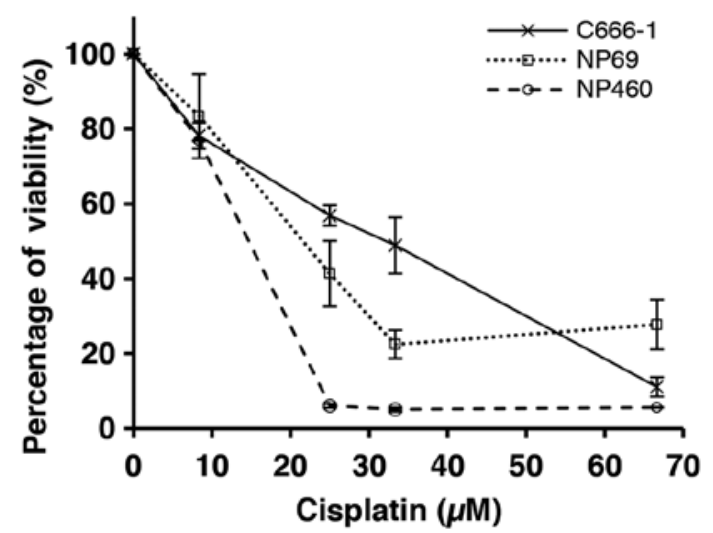

B

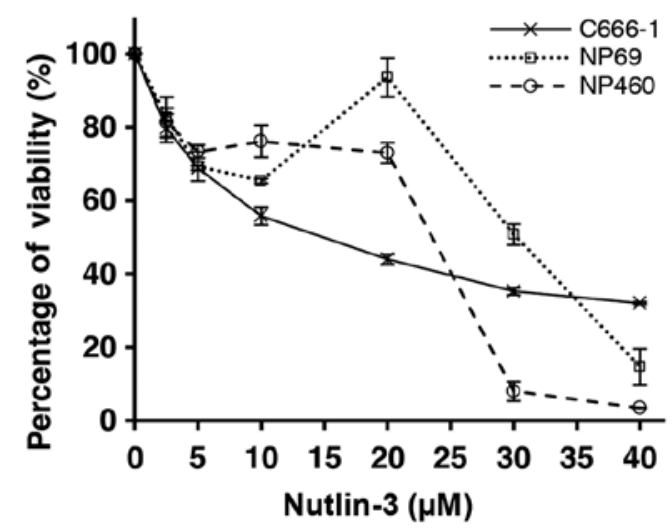

Figure 1. Growth-inhibitory effects of cisplatin and Nutlin-3 on NPC (C666-1) and NPE (NP69, NP460) cells. Cells were treated with increasing concentrations of (A) cisplatin $(0-66.64 \mu \mathrm{M})$ or $(\mathrm{B})$ Nutlin-3 $(0-40 \mu \mathrm{M})$ for $72 \mathrm{~h}$. Cell viability was determined by MTS viability assay. The average percentage of cell viability relative to the untreated and $0.1 \%$ DMSO-treated controls are shown.

Table I. Sensitivity of the NPC and NPE cell lines to cisplatin and Nutlin-3 as indicated by $\mathrm{IC}_{50} \pm \mathrm{SD}$ values.

\begin{tabular}{lcc}
\hline & \multicolumn{2}{c}{$\mathrm{IC}_{50}$ values $(\mu \mathrm{M})$} \\
\cline { 2 - 3 } Cell lines & Cisplatin & Nutlin-3 \\
\hline NP69 & $19.31 \pm 4.75$ & $31.69 \pm 2.54$ \\
NP460 & $14.18 \pm 2.97$ & $22.85 \pm 1.18$ \\
C666-1 & $32.07 \pm 4.18$ & $19.95 \pm 8.93$ \\
\hline
\end{tabular}

Representative $\mathrm{IC}_{50} \pm \mathrm{SD}$ values are the mean of three independent experiments with nine data points. $\mathrm{IC}_{50}$, half maximal $(50 \%)$ inhibitory concentration; SD, standard deviation.

the efficiency of anchorage-independent colony formation using soft agar colony formation assay. Cisplatin $(33.33 \mu \mathrm{M})$ and Nutlin-3 $(10 \mu \mathrm{M})$ significantly reduced the number of colonies formed when administered alone as compared to the untreated and DMSO-treated controls. When the two treatments were combined, the numbers of colonies formed were markedly reduced (Fig. 2C) and the sizes of colonies were also significantly smaller (Fig. 2D). This further supports the earlier observation that Nutlin-3 sensitizes C666-1 cells to the cytotoxic effect of cisplatin and suppresses colony formation.

Nutlin-3 activates the p53 pathway in wt p53 NPC cells. The effects of Nutlin-3 on the p53 pathway were investigated on NPC C666-1 and HK1 cells harboring wt and mutant p53, respectively. Investigation was also performed in parallel with colorectal (HCT116) and breast (MDA-MB-231) cancer cells harboring wt and mutant $\mathrm{p} 53$, respectively. The expression levels of cellular proteins p53, p21Waf1/Cip1 and Mdm2 are shown in Fig. 3A. Upon treatment of C666-1 or HCT116 cells with Nutlin-3, p53 was activated and this significantly induced the expression of $\mathrm{p} 21$ protein and to a lesser extent, Mdm2 protein. The effects of Nutlin-3 were stronger in cells bearing wt $\mathrm{p} 53$ compared to cells with mutated p53 (HK1 and MDA-MB-231 cells). These findings suggest that Nutlin-3 activates the $\mathrm{p} 53$ pathway and induces upregulation of $\mathrm{p} 53, \mathrm{p} 21$ and $\mathrm{Mdm} 2$ in cells bearing wt $\mathrm{p} 53$.

Nutlin-3 activates the p53 pathway and exerts its cytotoxic effects on NPC cells in a p53-dependent manner. To verify that the cytotoxic effects of Nutlin-3 are mediated through the p53 pathway, we knocked down the p53 gene in the C666-1 cells using four different lentiviral-based shRNA constructs (p53si-E1, -E2, -C12 or -D3). The efficiency of knockdown was verified by examining the p53 protein expression level as shown in Fig. 3B; Vector-pLKO and NS were used as controls. Cells transduced with p53si-E2 and p53si-D3 constructs had $70(\mathrm{P}<0.05)$ and $90 \%(\mathrm{P}<0.005)$ knockdown of p53 protein expression, respectively; whereas, the control Vector-pLKO and NS had unaltered $\mathrm{p} 53$ protein expression. Next, the effects of Nutlin-3 on the expression of p53-related proteins following the knockdown of $\mathrm{p} 53$ protein in the lenti-sh $\Delta \mathrm{p} 53$-transduced cells were studied. When the parental C666-1 cells were exposed to $10 \mu \mathrm{M}$ Nutlin-3, increased expression levels of p53, p21 and Mdm2 were observed in comparison to the untreated-parental C666-1 cells and control Vector-pLKO and NS (Fig. 3C). However, these effects were markedly inhibited in the p53-knockdown C666-1 cells transduced with the p53si-D3 or p53si-E2 construct. The percentages of viability relative to control Vector-pLKO and NS following treatment with Nutlin-3 are shown in Fig. 3D. Cells with p53 knockdown were found to be less sensitive to Nutlin-3 in comparison to the controls. Collectively, these findings suggest that the effect of Nutlin-3 on C666-1 cell viability is p53-dependent.

Nutlin-3 sensitizes NPC cells to cisplatin-induced apoptosis. As shown in Fig. 2, the combination of cisplatin and Nutlin-3 effectively impaired cell viability and markedly suppressed the tumorigenicity of C666-1 cells. We used high content imaging of Annexin V/PI-stained cells to determine the effects of the drug combination on induction of apoptosis (Fig. 4A and B). Treatment of C666-1 cells with cisplatin resulted in apoptosis. Apoptosis increased significantly in the cells treated with both cisplatin and Nutlin-3. These observations indicated that Nutlin-3 sensitized C666-1 cells to cisplatin and enhanced 
A

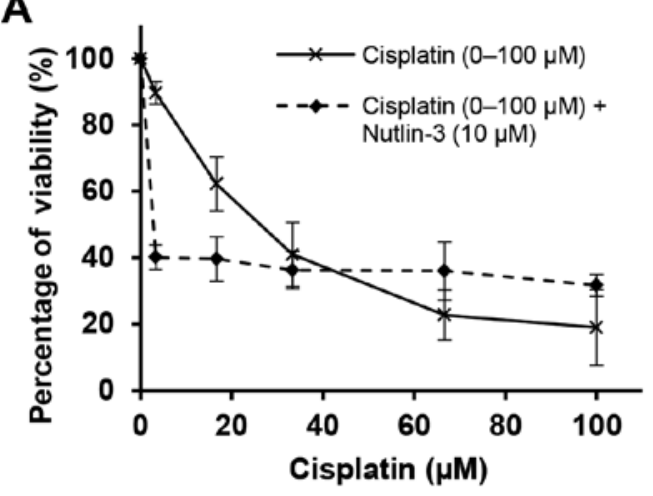

B

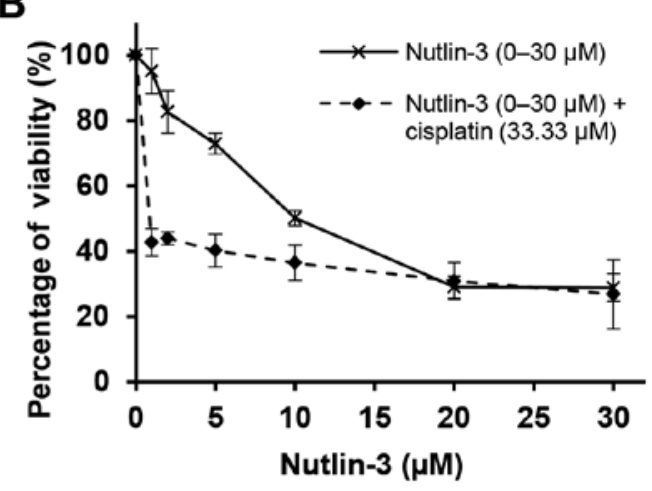

C
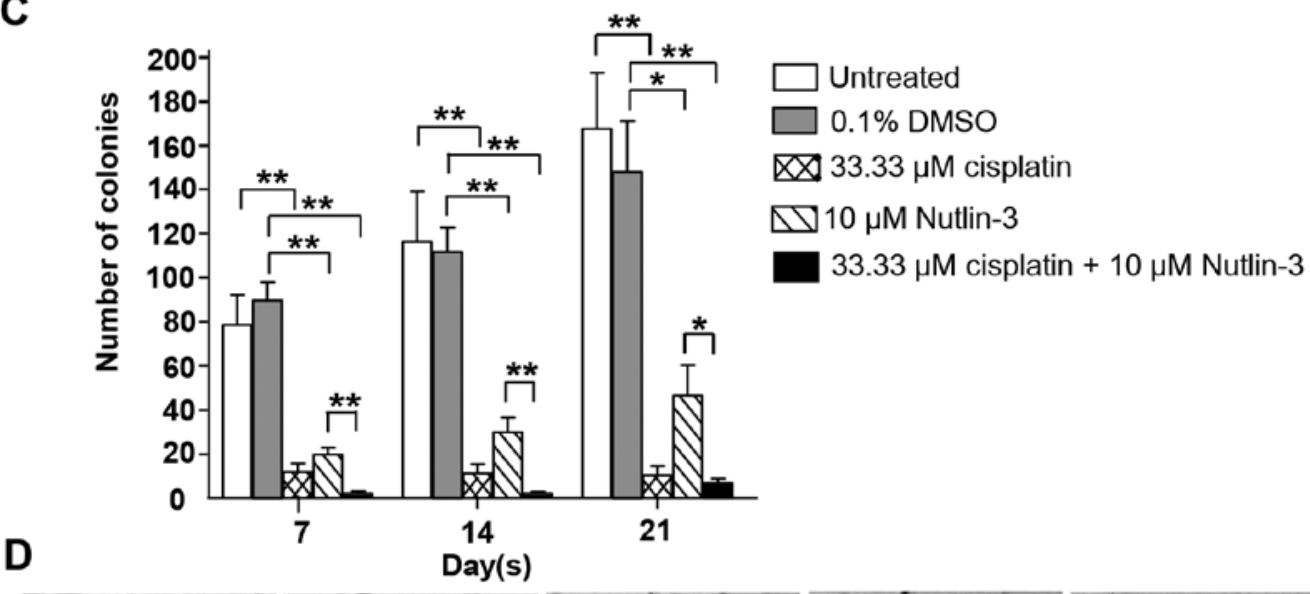

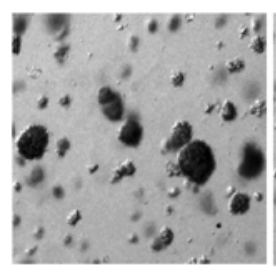

Untreated

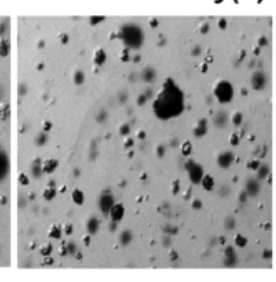

$0.1 \%$ DMSO

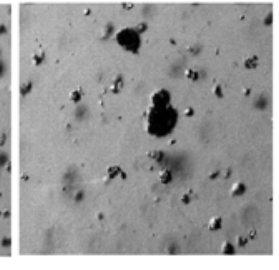

$33.33 \mu \mathrm{M}$ cisplatin

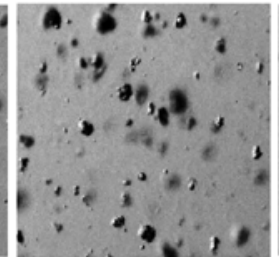

$10 \mu \mathrm{M}$ Nutlin-3

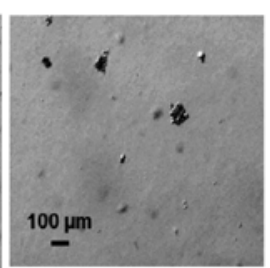

$33.33 \mu \mathrm{M}$ cisplatin

Figure 2. Nutlin-3 sensitizes C666-1 cells to the cytotoxic effect of cisplatin. The cell viability in response to (A) cisplatin alone (0-100 $\mu \mathrm{M})$ and in combination with $10 \mu \mathrm{M}$ Nutlin-3, and (B) Nutlin-3 alone (0-30 $\mu \mathrm{M})$ and in combination with $33.33 \mu \mathrm{M}$ cisplatin were assayed by MTS viability assay following $48 \mathrm{~h}$ of treatment. The graphs shown are representative average percentages of cell viability relative to the untreated and $0.1 \%$ DMSO-treated controls of three independent experiments with nine data points. (C) Effect of Nutlin-3 alone and in combination with cisplatin on colony formation of C666-1 cells. The plot shown is representative of two independent experiments carried out in triplicate. The untreated and $0.1 \%$ DMSO-treated cells were included as controls for cisplatin and Nutlin-3 treatments, respectively. Statistical analysis using the paired Student's t-test was performed to compare the difference in number of colonies scored on day 7, 14 and 21. ${ }^{*} \mathrm{P}<0.05$ and ${ }^{* *} \mathrm{P}<0.005$ compared to the control. (D) Morphological appearances of C666-1 cell colonies in soft agar cultures. The image of the colonies was captured on day 21 using an Olympus stereomicroscope at a magnification of $\times 2.5$ (scale bar, $100 \mu \mathrm{m}$ ).

apoptotic cell death. Next, we investigated whether Nutlin-3 activates other p53-mediated pro-apoptotic genes (Fig. 4C). Treatment with Nutlin-3 alone significantly upregulated BAX and PUMA protein expression. Similarly, the expression levels of p53, BAX and PUMA were further enhanced by the combination of cisplatin and Nutlin-3. Furthermore, the cleaved PARP level detected in the cells treated with the combination drugs was consistent with apoptosis. Collectively, these results suggest that Nutlin-3 sensitized the C666-1 cells to cisplatininduced apoptosis by modulating pro-apoptotic targets PUMA and BAX.

Extended treatment with Nutlin-3 results in reduced sensitivity without emergence of p53 mutation. p53 mutations are known to result in resistance to Nutlin-3 (36). To investigate whether Nutlin-3 induces the emergence of p53 mutant cells, C666-1 cells were treated with various concentrations of Nutlin-3 for extended periods (Fig. 5A). The Nutlin-3-treated NPC sublines showed a doubling in the $\mathrm{IC}_{50}$ value to Nutlin-3 when compared to the parental C666-1 cells, suggesting that the C666-1 cells were developing relative resistance to Nutlin-3 (Fig. 5B). Next, the Nutlin-3-resistant sublines were investigated for p53 status. The p53 gene sequence (spanning exon 2 to 11) of the investigated sublines was found to be wt compared to the parental C666-1 cells. These results indicate that, while extended treatment of NPC cells with Nutlin-3 resulted in decreased sensitivity to Nutlin-3, it was not associated with emergence of p53 mutations at the investigated doses and durations. 

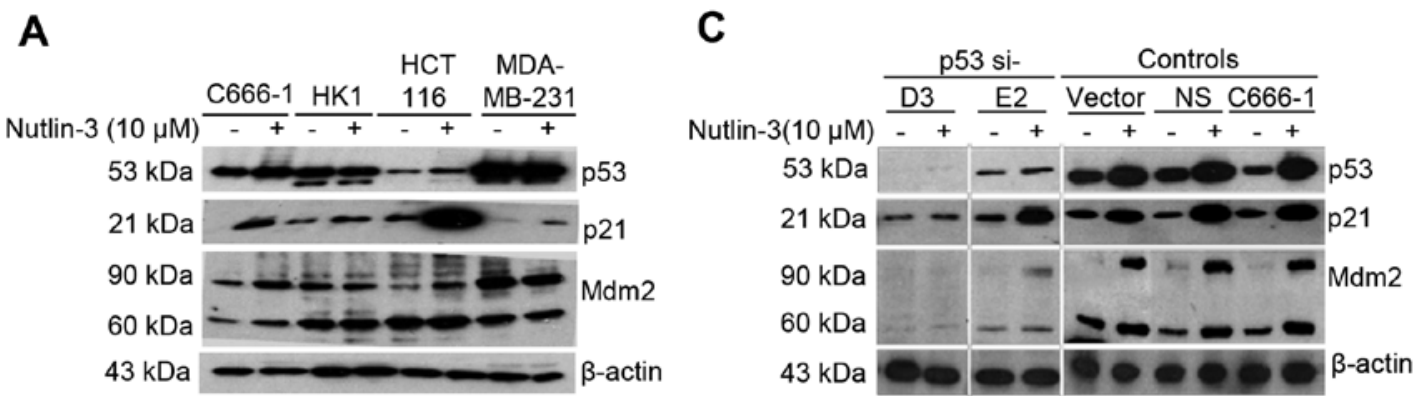

B

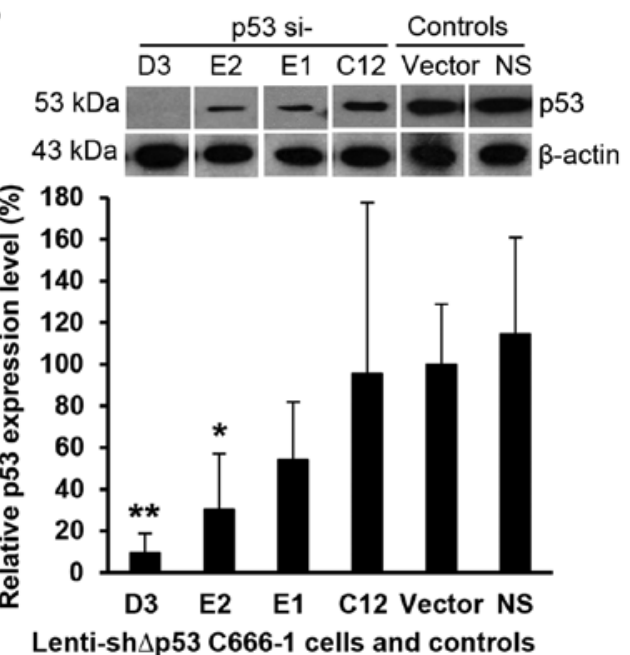

D

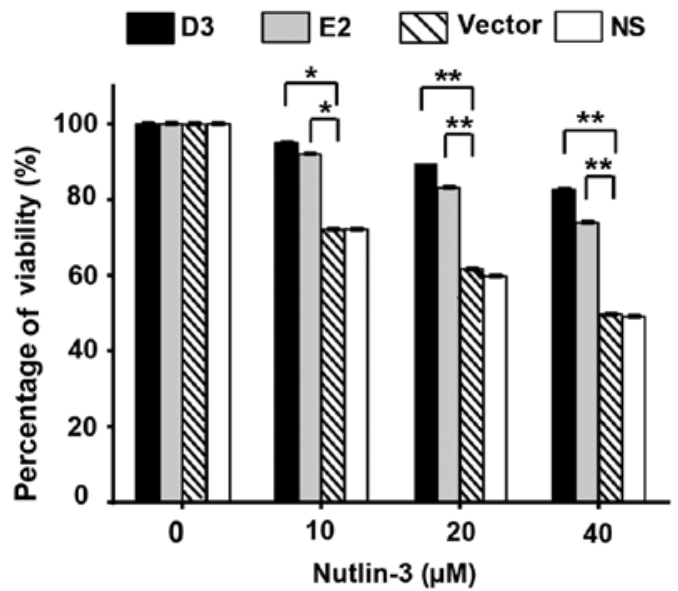

Figure 3. Nutlin-3 activates the p53 pathway, upregulating p53, p21 and Mdm2 in C666-1 cells in a p53-dependent manner. (A) The expression of p53-related proteins in cancer cells bearing wt p53 (C666-1 and HCT116) and mutant p53 (HK1 and MDA-MB-231) after being treated with $10 \mu \mathrm{M}$ Nutlin-3 (+) or 0.1\% DMSO (-) for $24 \mathrm{~h}$ are shown. $\beta$-actin is included as loading control. Nutlin-3 induced the upregulation of p53, p21 and Mdm2 proteins in the C666-1 and HCT116 cells bearing wt p53. (B) Establishment of p53 knockdown. p53-knockdown C666-1 cell sublines (lenti-sh $\Delta$ p53 C666-1) were generated using the lentiviral-based shRNA constructs p53si-E1, -E2, -C12 or -D3 in parallel with the control vector and NS. The efficiency of knockdown was verified at $96 \mathrm{~h}$ post transduction. The plot shown is representative of three independent experiments. The mean percentage of the relative p53 expression level was obtained by densitometric analysis when normalized to $\beta$-actin. Among the 4 constructs, the p53 protein level was most significantly reduced by p53si-D3 or p53si-E2 (C) The effects of Nutlin-3 on the expression of p53, p21 and Mdm2 proteins in the lenti-sh $\Delta$ p53 C666-1 cells. The lenti-sh $\Delta$ p53 C666-1 and control cells were treated with $10 \mu \mathrm{M}$ Nutlin-3 for $24 \mathrm{~h}$ prior to analysis. The attenuation of the activation of the p53 pathway was evident in cells transduced with p53si-D3 or p53si-E2 compared to the controls. (D) Growth inhibitory effect of Nutlin-3 on lenti-sh $\Delta$ p53 C666-1 cells. Cell viability responses to $0-40 \mu \mathrm{M}$ Nutlin-3 after a 72 -h treatment was quantified by MTS viability assay. The average percentage of cell viability relative to the $0.1 \%$ DMSO-treated vehicle control is shown for the lenti-sh $\Delta$ p53 C666-1 cells and controls. The plot shown is representative of mean values of three independent experiments with nine data points. ${ }^{*} \mathrm{P}<0.05$ and ${ }^{* *} \mathrm{P}<0.005$ compared to the vector. Vector, vector-pLKO; NS, non-specific.

\section{Discussion}

Targeting p53-Mdm2 interaction to reactivate p53 tumorsuppressing function is a promising cancer therapeutic strategy for tumors retaining wt p53 (37). The disruption of p53-Mdm2 interaction to reactivate the p53 pathway by smallmolecule inhibitor Nutlin-3 is restricted to tumors with wt p53 (14). Hence, in the present study, a prior verification of the p53 status of our NPC and NPE cell lines was performed. A base change of $\mathrm{G}>\mathrm{C}$ at codon 72 of the 4 th exon of the $\mathrm{p} 53$ gene, known as codon 72 polymorphism which encodes for variant amino acids: arginine (CGC) or proline (CCC) was previously reported (38). In the present study, we found the identical codon 72 polymorphism in the p53 gene of our NP69, NP460 and C666-1 cell lines. This polymorphism is common in the wt p53 gene and has been detected in lung cancer (39), teratoma (40) and esophageal squamous cell carcinoma (41). As expected, our HK1 cell line had a homozygous point mutation
$\mathrm{C}>\mathrm{G}$ at codon 130 of the 5 th exon of $\mathrm{p} 53$ gene, substituting leucine (CTC) with valine (GTC), similar to a previous report (42).

The present study demonstrated that Nutlin-3 sensitized NPC cells to cisplatin-induced cytotoxicity in a p53-dependent manner. Nutlin-3 reduced the viability and colony formation of wt p53 NPC cells. Furthermore, Nutlin-3 significantly reduced the viability of NPC, but not NPE cells at a concentration of 10-20 $\mu \mathrm{M}$, consistent with recent studies that showed that Nutlin-3 was not toxic to normal cells at the concentrations of $10-20 \mu \mathrm{M}(18,43)$.

Nutlin-3 activated p53 and upregulated p21 and Mdm2 in the C666-1 and HCT116 cancer cells retaining wt p53, but not in the HK1 and MDA-MB-231 cancer cells lacking wt $\mathrm{p} 53$. These findings are in concordance with previous reports that Nutlin-3 induced apoptosis in p53 wt, but not p53 mutant cancer cells $(14,16,26,44)$. However, Nutlin-3 has also been reported to act via p53-independent pathways (45). 
A

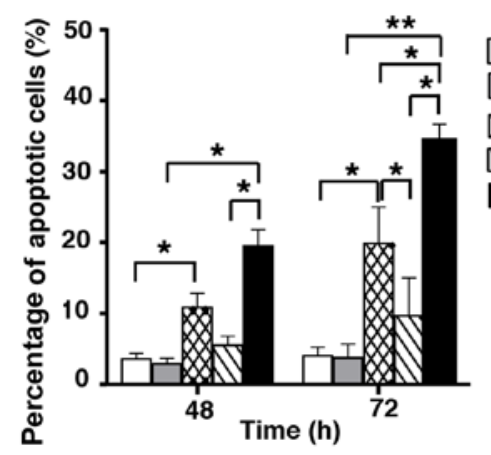

B

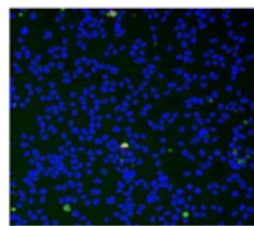

Untreated

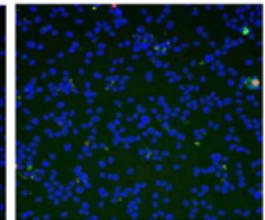

$0.1 \%$ DMSO
Untreated

$0.1 \%$ DMSO

$\triangle 33.33 \mu \mathrm{M}$ cisplatin

$\triangle 10 \mu \mathrm{M}$ Nutlin-3

33.33 $\mu \mathrm{M}$ cisplatin

$+10 \mu \mathrm{M}$ Nutlin-3

C

$53 \mathrm{kDa}-\mathrm{p} 53$

$21 \mathrm{kDa}-2 \mathrm{l}-\mathrm{p} 21$

$90 \mathrm{kDa}-0 \circlearrowright-\mathrm{Mdm} 2$

$60 \mathrm{kDa}$

$20 \mathrm{kDa}-\mathrm{BAX}$

$23 \mathrm{kDa}$ PUMA

$28 \mathrm{kDa}-\infty \mathrm{Bcl} 2$

$116 \mathrm{kDa}$

$89 \mathrm{kDa}$

$45 \mathrm{kDa}=-\beta-\beta$-actin

Cisplatin $(33.33 \mu \mathrm{M}) \quad-\quad+\quad-\quad+$

Nutlin-3 (10 $\mu \mathrm{M})$

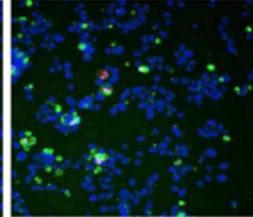

$33.33 \mu \mathrm{M}$ cisplatin

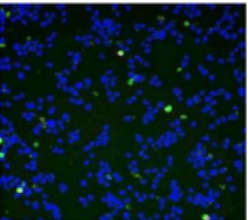

$10 \mu \mathrm{M}$ Nutlin-3

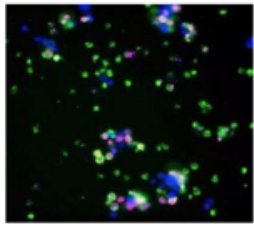

$33.33 \mu \mathrm{M}$ cisplatin $+10 \mu \mathrm{M}$ Nutlin-3

Figure 4. Nutlin-3 sensitizes C666-1 cells to cisplatin-induced apoptosis. (A) High content analysis of Annexin V-FITC/PI-stained cells to measure apoptosis induced by cisplatin and/or Nutlin-3 in C666-1 cells. The cells were treated with $33.33 \mu \mathrm{M}$ cisplatin and/or $10 \mu \mathrm{M}$ Nutlin-3 for 48 and $72 \mathrm{~h}$ prior to analysis. The untreated and $0.1 \%$ DMSO-treated cells served as non-treated controls for cisplatin and Nutlin-3, respectively. Student's t-test was performed to compare the difference in the apoptotic cell score. ${ }^{*} \mathrm{P}<0.05$ and ${ }^{* *} \mathrm{P}<0.005$ compared to the control. (B) Representative high content images of C666-1 cells stained with Annexin V-FITC for apoptosis (green), PI for viability (red) and Hoechst 33342 as counterstaining (blue) are shown. Imaging was conducted at $72 \mathrm{~h}$ post-treatment at magnification of x20. Annexin V(+)/PI(-) and Annexin V(+)/PI(+) cells were defined as apoptotic cells. (C) The effects of Nutlin-3 on the expression of apoptosis-related proteins in cisplatin-treated C666-1 cells. C666-1 cells were treated with cisplatin and/or Nutlin-3 (+) or 0.1\% DMSO (-) for $24 \mathrm{~h}$ prior to analysis. The levels of p53 target apoptosis-related proteins are shown. $\beta$-actin was probed to monitor protein loading. Treatment of C666-1 cells with Nutlin-3 resulted in induction of p53 target apoptosis-related proteins p21, Mdm2, BAX and PUMA as compared to the vehicle control. FITC, fluorescein isothiocyanate; PI, propidium iodide.

A

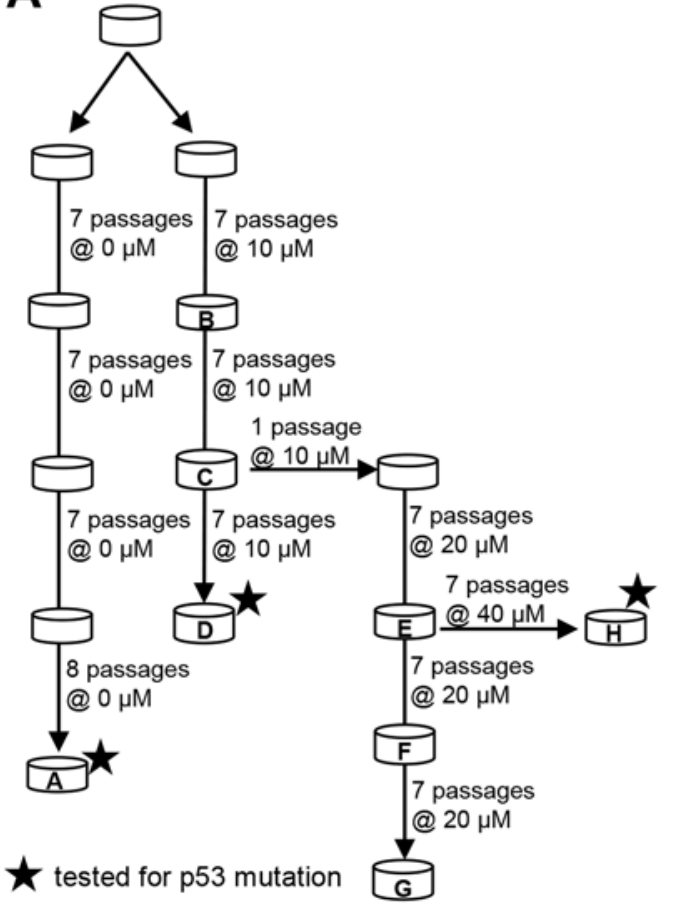

B

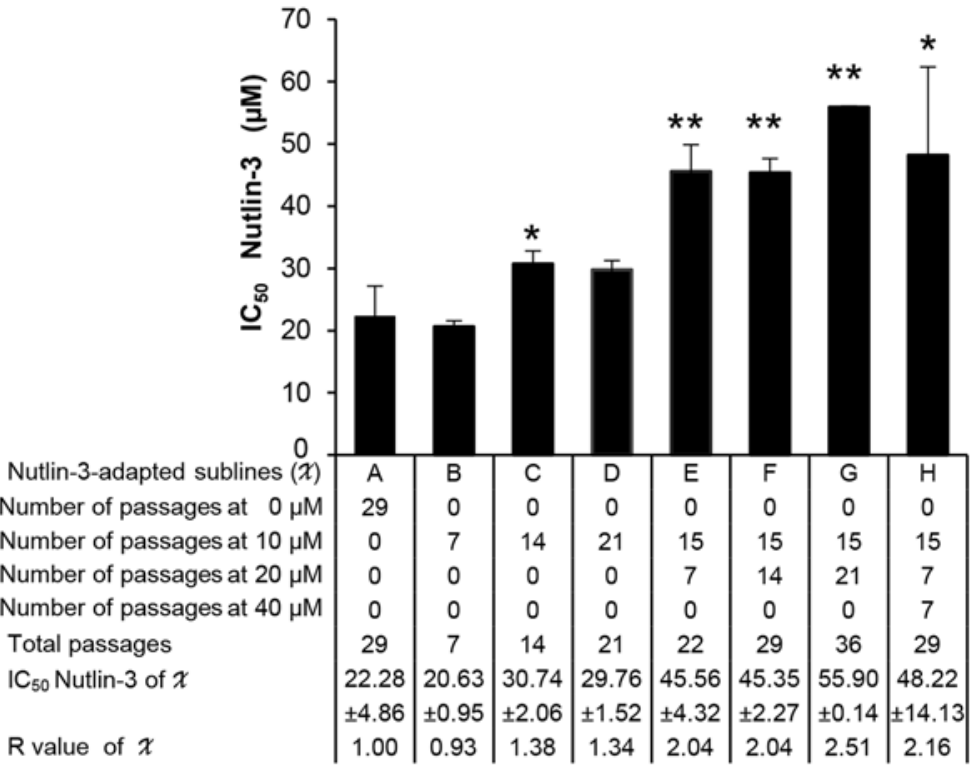

Figure 5. Extended treatment with Nutlin-3 results in reduced sensitivity without emergence of p53 mutations. (A) The diagram shown is the experimental design to establish Nutlin-3-adapted NPC cell sublines. Nutlin-3-adapted C666-1 cell sublines were established by treatment of C666-1 cells with 10 to $40 \mu \mathrm{M}$ Nutlin-3 over a period of up to six months. The sublines tested for p53 mutations are labeled ( $\star$ ). (B) Sensitivity of Nutlin-3-adapted C666-1 sublines to Nutlin-3 indicated by the concentration that inhibits cell viability by $50 \%\left(\mathrm{IC}_{50}\right)$ following a 72 -h treatment as quantified by MTS viability assay. The plot shown is representative mean $\mathrm{IC}_{50}$ values of three independent experiments carried out in triplicate. ${ }^{*} \mathrm{P}<0.05$ and ${ }^{* * *} \mathrm{P}<0.005$ compared to the parental control. $(\mathcal{X})$, Nutlin-3 adapted C666-1 sublines; $\mathrm{R}$, resistance index indicates the degree of acquired resistance. 
Nevertheless, suppression of endogenous p53 by lentiviralbased shRNA inhibited the effects of Nutlin-3 on C666-1 cells, thus indicating that the effects of Nutlin-3 on C666-1 cells were p53-dependent.

The anti-proliferative activity of cisplatin on NPC cells was found to be enhanced when combined with Nutlin-3. Our data indicate that Nutlin-3 sensitized the NPC cells to cisplatin-induced apoptosis. Similarly, these synergistic activities have been reported in neuroblastoma (46), gastric cancer (47) and ovarian cancer cells (48) and testicular germ cell tumors (49). Collectively, the enhancement of cisplatininduced apoptotic cell death of NPC cells by Nutlin-3 was evidenced by the upregulation of p53, p21, Mdm2, BAX and PUMA expressions. These findings are in agreement with the notion that $\mathrm{p} 53$ promotes apoptosis by activating p53 pro-apoptotic targets.

Long-term treatment of cancer cells with increasing concentrations of Nutlin-3 could result in the emergence of Nutlin-3-resistant p53-mutated cells (36). In the present study, we found that although long-term exposure of wt p53 C666-1 cells to $10-40 \mu \mathrm{M}$ Nutlin-3 in stepwise dose increments resulted in reduced sensitivity to Nutlin-3, the treatment did not result in the emergence of p53-mutated cells. This finding suggests that the acquisition of Nutlin-3 resistance in these cells could be due to other mechanisms. The reduction in sensitivity to Nutlin-3 in long-term treatment suggests that optimization in the clinical dose could be important to enhance the efficacy of Nutlin-3. However, such clinical implications have yet to be investigated fully.

In conclusion, we present evidence that Nutlin-3 is an effective p53 activator in NPC cells. Nutlin-3 was more toxic to NPC cells compared to non-malignant cells. The effects of Nutlin-3 on NPC cells were p53-dependent. In combination with cisplatin, Nutlin-3 promoted apoptosis induction in the NPC cells expressing wt p53. Collectively, the present study suggests that the potential use of inhibitors of p53-Mdm2 interaction should be explored further for the treatment of NPC.

\section{Acknowledgements}

The authors would like to thank the Director General of Health Malaysia for his permission to publish this paper and the Director of the Institute for Medical Research for her support. We would like to thank Professor Kwok-Wai Lo (Chinese University of Hong Kong) and Professor George Tsao (University of Hong Kong) for kindly providing the C666-1 and HK1 cells, respectively. The present study was supported and funded by the Ministry of Health Malaysia.

\section{References}

1. Pathmanathan R, Prasad U, Chandrika G, Sadler R, Flynn K and Raab-Traub N: Undifferentiated, nonkeratinizing, and squamous cell carcinoma of the nasopharynx. Variants of Epstein-Barr virus-infected neoplasia. Am J Pathol 146: 1355-1367, 1995.

2. Devi BC, Pisani P, Tang TS and Parkin DM: High incidence of nasopharyngeal carcinoma in native people of Sarawak, Borneo Island. Cancer Epidemiol Biomarkers Prev 13: 482-486, 2004.

3. Zeng MS and Zeng YX: Pathogenesis and etiology of nasopharyngeal carcinoma. In: Nasopharyngeal Cancer Medical Radiology. Springer Berlin, Heidelberg, pp9-25, 2010.
4. Khoo AS and Pua KC: Diagnosis and clinical evaluation of nasopharyngeal carcinoma. In: Nasopharyngeal Carcinoma: Keys for Translational Medicine and Biology. Landes Bioscience and Springer Science: Business Media, New York, NY, pp1-9, 2013.

5. Pua KC, Khoo AS, Yap YY, Subramaniam SK, Ong CA, Gopala Krishnan G, Shahid H and The Malaysian Nasopharyngeal Carcinoma Study Group: Nasopharyngeal Carcinoma Database. Med J Malaysia 63 (Suppl C): 59-62, 2008.

6. Lee AW, Lin JC and Ng WT: Current management of nasopharyngeal cancer. Semin Radiat Oncol 22: 233-244, 2012.

7. Zhang L, Chen QY, Liu H, Tang LQ and Mai HQ: Emerging treatment options for nasopharyngeal carcinoma. Drug Des Devel Ther 7: 37-52, 2013

8. Chee Ee Phua V, Loo WH, Yusof MM, Wan Ishak WZ, Tho LM and Ung NM: Treatment outcome for nasopharyngeal carcinoma in University Malaya Medical Centre from 2004-2008. Asian Pac J Cancer Prev 14: 4567-4570, 2013.

9. Razak AR, Siu LL, Liu FF, Ito E, O'Sullivan B and Chan K: Nasopharyngeal carcinoma: The next challenges. Eur J Cancer 46: 1967-1978, 2010.

10. Tuan JK, Ha TC, Ong WS, Siow TR, Tham IW, Yap SP, Tan TW, Chua ET, Fong KW and Wee JT: Late toxicities after conventional radiation therapy alone for nasopharyngeal carcinoma. Radiother Oncol 104: 305-311, 2012.

11. Brown CJ, Lain S, Verma CS, Fersht AR and Lane DP: Awakening guardian angels: Drugging the p53 pathway. Nat Rev Cancer 9: 862-873, 2009.

12. Michael D and Oren M: The p53-Mdm2 module and the ubiquitin system. Semin Cancer Biol 13: 49-58, 2003.

13. Fuchs SY, Adler V, Buschmann T, Wu X and Ronai Z: Mdm2 association with p53 targets its ubiquitination. Oncogene 17: 2543-2547, 1998

14. Vassilev LT, Vu BT, Graves B, Carvajal D, Podlaski F, Filipovic Z, Kong N, Kammlott U, Lukacs C, Klein C, et al: In vivo activation of the p53 pathway by small-molecule antagonists of MDM2. Science 303: 844-848, 2004.

15. Wiman KG: Strategies for therapeutic targeting of the p53 pathway in cancer. Cell Death Differ 13: 921-926, 2006.

16. Kojima K, Konopleva M, Samudio IJ, Shikami M, CabreiraHansen M, McQueen T, Ruvolo V, Tsao T, Zeng Z, Vassilev LT, et al: MDM2 antagonists induce p53-dependent apoptosis in AML: Implications for leukemia therapy. Blood 106: 3150-3159, 2005.

17. Saddler C, Ouillette P, Kujawski L, Shangary S, Talpaz M, Kaminski M, Erba H, Shedden K, Wang S and Malek SN: Comprehensive biomarker and genomic analysis identifies p53 status as the major determinant of response to MDM2 inhibitors in chronic lymphocytic leukemia. Blood 111: 1584-1593, 2008.

18. Stühmer T, Chatterjee M, Hildebrandt M, Herrmann P, Gollasch H, Gerecke C, Theurich S, Cigliano L, Manz RA, Daniel PT, et al: Nongenotoxic activation of the p53 pathway as a therapeutic strategy for multiple myeloma. Blood 106: 3609-3617, 2005.

19. Ye F, Lattif AA, Xie J, Weinberg A and Gao S: Nutlin-3 induces apoptosis, disrupts viral latency and inhibits expression of angiopoietin-2 in Kaposi sarcoma tumor cells. Cell Cycle 11: 1393-1399, 2012.

20. Müller CR, Paulsen EB, Noordhuis P, Pedeutour F, Saeter G and Myklebost O: Potential for treatment of liposarcomas with the MDM2 antagonist Nutlin-3A. Int J Cancer 121: 199-205, 2007.

21. Miyachi M, Kakazu N, Yagyu S, Katsumi Y, Tsubai-Shimizu S, Kikuchi K, Tsuchiya K, Iehara T and Hosoi H: Restoration of p53 pathway by nutlin-3 induces cell cycle arrest and apoptosis in human rhabdomyosarcoma cells. Clin Cancer Res 15: 4077-4084, 2009.

22. Sonnemann J, Palani CD, Wittig S, Becker S, Eichhorn F, Voigt A and Beck JF: Anticancer effects of the p53 activator nutlin-3 in Ewing's sarcoma cells. Eur J Cancer 47: 1432-1441, 2011.

23. Hori T, Kondo T, Kanamori M, Tabuchi Y, Ogawa R, Zhao QL, Ahmed K, Yasuda T, Seki S, Suzuki K, et al: Nutlin-3 enhances tumor necrosis factor-related apoptosis-inducing ligand (TRAIL)-induced apoptosis through up-regulation of death receptor 5 (DR5) in human sarcoma HOS cells and human colon cancer HCT116 cells. Cancer Lett 287: 98-108, 2010.

24. Koster R, Timmer-Bosscha H, Bischoff R, Gietema JA and de Jong S: Disruption of the MDM2-p53 interaction strongly potentiates p53-dependent apoptosis in cisplatin-resistant human testicular carcinoma cells via the Fas/FasL pathway. Cell Death Dis 2: e148, 2011. 
25. Tovar C, Rosinski J, Filipovic Z, Higgins B, Kolinsky K, Hilton $\mathrm{H}$ Zhao X, Vu BT, Qing W, Packman K, et al: Small-molecule MDM2 antagonists reveal aberrant p53 signaling in cancer: Implications for therapy. Proc Natl Acad Sci USA 103: 1888-1893, 2006.

26. Van Maerken T, Ferdinande L, Taildeman J, Lambertz I, Yigit N, Vercruysse L, Rihani A, Michaelis M, Cinatl J Jr, Cuvelier CA, et al: Antitumor activity of the selective MDM2 antagonist nutlin-3 against chemoresistant neuroblastoma with wild-type p53. J Natl Cancer Inst 101: 1562-1574, 2009.

27. Khoo KH, Verma CS and Lane DP: Drugging the p53 pathway: Understanding the route to clinical efficacy. Nat Rev Drug Discov 13: 217-236, 2014

28. Effert P, McCoy R, Abdel-Hamid M, Flynn K, Zhang Q, Busson P, Tursz T, Liu E and Raab-Traub N: Alterations of the p53 gene in nasopharyngeal carcinoma. J Virol 66: 3768-3775, 1992.

29. Hoe SL, Lee ES, Khoo AS and Peh SC: p53 and nasopharyngeal carcinoma: A Malaysian study. Pathology 41: 561-565, 2009.

30. Chang KP, Hao SP, Lin SY, Tsao KC, Kuo TT, Tsai MH, Tseng CK and Tsang NM: A lack of association between p53 mutations and recurrent nasopharyngeal carcinomas refractory to radiotherapy. Laryngoscope 112: 2015-2019, 2002.

31. Hui AB, Lo KW, Leung SF, Teo P, Fung MK, To KF, Wong N, Choi PH, Lee JC and Huang DP: Detection of recurrent chromosomal gains and losses in primary nasopharyngeal carcinoma by comparative genomic hybridisation. Int J Cancer 82: 498-503, 1999.

32. Zhang ZC, Fu S, Wang F, Wang HY, Zeng YX and Shao JY: Oncogene mutational profile in nasopharyngeal carcinoma. Onco Targets Ther 7: 457-467, 2014.

33. Liu MT, Chang YT, Chen SC, Chuang YC, Chen YR, Lin CS and Chen JY: Epstein-Barr virus latent membrane protein 1 represses p53-mediated DNA repair and transcriptional activity. Oncogene 24: 2635-2646, 2005.

34. Pan JJ, Zhang SW, Chen CB, Xiao SW, Sun Y, Liu CQ, Su X, $\mathrm{Li}$ DM, Xu G, Xu B, et al: Effect of recombinant adenovirus-p53 combined with radiotherapy on long-term prognosis of advanced nasopharyngeal carcinoma. J Clin Oncol 27: 799-804, 2009.

35. Weinrib L, Li JH, Donovan J, Huang D and Liu FF: Cisplatin chemotherapy plus adenoviral p53 gene therapy in EBV-positive and -negative nasopharyngeal carcinoma. Cancer Gene Ther 8: 352-360, 2001

36. Michaelis M, Rothweiler F, Barth S, Cinatl J, van Rikxoort M, Löschmann N, Voges Y, Breitling R, von Deimling A, Rödel F, et al: Adaptation of cancer cells from different entities to the MDM2 inhibitor nutlin-3 results in the emergence of p53-mutated multi-drug-resistant cancer cells. Cell Death Dis 2: e243, 2011.
37. Shangary S and Wang S: Small-molecule inhibitors of the MDM2-p53 protein-protein interaction to reactivate p53 function: A novel approach for cancer therapy. Annu Rev Pharmacol Toxicol 49: 223-241, 2009.

38. Matlashewski GJ, Tuck S, Pim D, Lamb P, Schneider J and Crawford LV: Primary structure polymorphism at amino acid residue 72 of human p53. Mol Cell Biol 7: 961-963, 1987.

39. Fan R, Wu MT, Miller D, Wain JC, Kelsey KT, Wiencke JK and Christiani DC: The p53 codon 72 polymorphism and lung cancer risk. Cancer Epidemiol Biomarkers Prev 9: 1037-1042, 2000.

40. Udin N, Ahmad KA, Ahmad F, Omar E, Aziz ME, Kumar R and Abdullah JM: Molecular genetic analysis of a suprasellar immature teratoma : Mutation of exon 4 p53 gene. Malays J Med Sci 15: 43-46, 2008

41. Yang W, Zhang Y, Tian X, Ning T and Ke Y: p53 Codon 72 polymorphism and the risk of esophageal squamous cell carcinoma. Mol Carcinog 47: 100-104, 2008.

42. Spruck CH III, Tsai YC, Huang DP, Yang AS, Rideout WM III, Gonzalez-Zulueta M, Choi P, Lo KW, Yu MC and Jones PA: Absence of p53 gene mutations in primary nasopharyngeal carcinomas. Cancer Res 52: 4787-4790, 1992.

43. Jiang M, Pabla N, Murphy RF, Yang T, Yin XM, Degenhardt K, White $\mathrm{E}$ and Dong Z: Nutlin-3 protects kidney cells during cisplatin therapy by suppressing Bax/Bak activation. J Biol Chem 282: 2636-2645, 2007

44. Chang LJ and Eastman A: Differential regulation of p21 (waf1) protein half-life by DNA damage and Nutlin-3 in p53 wild-type tumors and its therapeutic implications. Cancer Biol Ther 13: 1047-1057, 2012

45. Valentine JM, Kumar S and Moumen A: A p53-independent role for the MDM2 antagonist Nutlin-3 in DNA damage response initiation. BMC Cancer 11: 79, 2011

46. Barbieri E, Mehta P, Chen Z, Zhang L, Slack A, Berg S and Shohet JM: MDM2 inhibition sensitizes neuroblastoma to chemotherapy-induced apoptotic cell death. Mol Cancer Ther 5: 2358-2365, 2006

47. Endo S, Yamato K, Hirai S, Moriwaki T, Fukuda K, Suzuki H, Abei M, Nakagawa I and Hyodo I: Potent in vitro and in vivo antitumor effects of MDM2 inhibitor nutlin-3 in gastric cancer cells. Cancer Sci 102: 605-613, 2011.

48. Mir R, Tortosa A, Martinez-Soler F, Vidal A, Condom E, Pérez-Perarnau A, Ruiz-Larroya T, Gil J and Giménez-Bonafé P: $\mathrm{Mdm} 2$ antagonists induce apoptosis and synergize with cisplatin overcoming chemoresistance in TP53 wild-type ovarian cancer cells. Int J Cancer 132: 1525-1536, 2013.

49. Bauer S, Mühlenberg T, Leahy M, Hoiczyk M, Gauler T, Schuler M and Looijenga L: Therapeutic potential of Mdm2 inhibition in malignant germ cell tumours. Eur Urol 57: 679-687, 2010. 